\title{
Nonlinearities, Dynamics, and Fractals
}

(6th Annual Meeting of ENGADYN, Tüchersfeld, September 23-26, 1996)

In 1990, annual meetings named "ENGADYN" were constituted, aiming at intensifying the international and interdisciplinary cooperation in the fields of nonlinearities, dynamics and fractals. Selected contributions to these meetings were published in Z. Naturforsch. 48a, 619-678 (1993), 49a, 831-837 (1994) and 50a, 1089-1150 (1995). Corresponding contributions to the meeting in September 1996 are presented in the following.

The topics concern: spatio-temporal phenomena such as breathers and frequency clustering in coupled systems and the relation between time delay systems and partial differential equations; problems of time reversibility with respect to computability and aspects of micro- and macro-systems; the statistics of traffic flow data; statistical approaches to fractals and whispering in the Bunimovich stadium. With these contributions we intend to reflect the common spirit and the controversal discussions occupying our group. 